\title{
Modelling the Effects of Vertical Transmission in Mosquito and the Use of Imperfect Vaccine on Chikungunya Virus Transmission Dynamics
}

\author{
Martins O. Onuorah ${ }^{1}$, Emmanuel I. Obi ${ }^{2}$, Bala G. Babangida ${ }^{3}$ \\ ${ }^{1}$ Department of Physical Sciences, Kampala International University, Kampala, Uganda \\ ${ }^{2}$ Department of Science Laboratory Technology, Federal Polytechnic Nasarawa, Nasarawa, Nigeria \\ ${ }^{3}$ Department of Mathematics and Statistics, Islamic University in Uganda, Mbale, Uganda \\ Email: ^martins.onuorah@kiu.ac.ug, eobi39@yahoo.com, rumaya2011@gmail.com
}

How to cite this paper: Onuorah, M.O., Obi, E.I. and Babangida, B.G. (2019) Modelling the Effects of Vertical Transmission in Mosquito and the Use of Imperfect Vaccine on Chikungunya Virus Transmission Dynamics. Applied Mathematics, 10, 245-267. https://doi.org/10.4236/am.2019.104019

Received: March 8, 2019

Accepted: April 26, 2019

Published: April 29, 2019

Copyright (c) 2019 by author(s) and Scientific Research Publishing Inc. This work is licensed under the Creative Commons Attribution International License (CC BY 4.0).

http://creativecommons.org/licenses/by/4.0/

\section{Open Access}

\begin{abstract}
In this paper, a deterministic mathematical model for Chikungunya virus (Chikv) transmission and control is developed and analyzed to underscore the effect of vaccinating a proportion of the susceptible human, and vertical transmission in mosquito population. The disease free, and endemic equilibrium states were obtained and the conditions for the local and global stability or otherwise were given. Sensitivity analysis of the effective reproductive number, $R_{c}$ (the number of secondary infections resulting from the introduction of a single infected individual into a population where a proportion is fairly protected) shows that the recruitment rate of susceptible mosquito $\left(\Lambda_{M}\right)$ and the proportion of infectious new births from infected mosquito ( $\beta$ ) are the most sensitive parameters. Bifurcation analysis of the model using center manifold theory reveals that the model undergoes backward bifurcation (coexistence of disease free and endemic equilibrium when $R_{C}<1$ ). Numerical simulation of the model shows that vaccination of susceptible human population with imperfect vaccine will have a positive impact and that vertical transmission in mosquito population has a negligible effect. To the best of our knowledge, our model is the first to incorporate vaccinated human compartment and vertical transmission in (Chikv) model.
\end{abstract}

\section{Keywords}

Chikungunya Virus, Stability, Equilibrium, Vaccination, Endemic

\section{Introduction}

Chikungunya is a mosquito-borne viral disease that was first observed in Tanza- 
nia in 1952 [1]. In 1964, there was epidemic of Chikungunya in Vellore, Calcutta and Maharashtra state/provinces of India [2]. Ibadan, South Western Nigeria witnessed an epidemic of Chikungunya virus in 1969 when the virus was isolated from 49 patients [3]. The disease has been identified in over 60 countries in Asia, Africa, Europe and America, and the name describes the stooping appearance of the sufferers [4]. It is an RNA virus that belongs to the alphavirus genus and the family [5]. The symptoms include abrupt onset of fever accompanied by joint pain, muscle pain headache, nausea and rash [6]. Occasionally the infection may go unrecognized or be misdiagnosed and could be acute, sub-acute and chronic.

In recent years, the virus has risen from relatively obscurity to become a global public health menace affecting millions of persons throughout the tropical and subtropical regions of the world and as such has also become a frequent cause of travel associated febrile illness [7]. The virus is transmitted through the bite of female Aedes aegypti and Aedes albopictus mosquitoes. Aedes aegypti breeds in the ubiquitous small pools of water found around human habitation [8]. Unlike Aedes aegypti which exists in tropical and subtropical area, Aedes albopictus can also thrive in temperate regions, thus potentially introducing Chikungunya to new ecological niche [9]. These species of mosquitoes are found biting throughout the daylight hours. Mother to child transmission of Chikungunya virus has been reported [10].

Diagnosis is by confirming the presence of anti-Chikungunya antibody in the patient. At the moment, there is no vaccine or treatment for the disease. Protection is by covering of exposed skin with long pants and long sleeved shirts, insect repellents and insecticide treated mosquito nets. Since the beginning of the 19th century, mathematical model has become a veritable tool in the study of vector-borne diseases [11] [12] [13]. For (Chikv), we cite the following work, Dumont and Domerg [14], propose a model, including human and mosquito compartments that are associated with the time course of the first epidemic of Chikungunya in Reunion Island. Using entomological results, they investigated the links between the episode of 2005 and the outbreak of 2006. Moulay, Azziz and Cadivel [15], developed a Chikungunya transmission model for the spread of the epidemic in both humans and mosquitoes, the model involves a temporal dynamics of vector (Aedes albopictus), depending on climatic factors. In the study, they provided estimates of the transmission potential of the virus and assessed the efficacy of the measures undertaken by public health authorities to control the epidemic spread in Italy. Ruiz et al. [16], analyzed the potential risk of Chikungunya introduction into the US, their study combines a climate-based mosquito population dynamics stochastic model with an epidemiological model to identify temporal windows that have epidemic risk.

Pongsumpun and Sangsawang [17], model studied theoretically an age-structured model for Chikungunya involving juvenile and adult human populations, giving conditions for the disease-free and endemic states respectively. They also sug- 
gested alternative way for controlling the 8disease. Yakob and Clements [18], analysed a simple, deterministic mathematical model for the transmission of the virus between humans and mosquitoes. They fitted the model to the large Reunion epidemic data and estimated the type reproduction number for Chikungunya, their model provided a close approximation of both the peak incidence of the outbreak and the final epidemic size.

In this work, we proposed a deterministic mathematical model for the spread, and control of Chikv. Our model attempt to bridge identified gaps in the works cited above. Specifically, our model incorporated an imperfect vaccinated human compartment and vertical transmission in the mosquito population.

\section{Model Formulation}

The chic model is represented by nine non-linear ordinary differential equation consisting of human-sub population and mosquito sub-population. The human sub-population is divided into; susceptible human $S_{H}$, vaccinated human $V_{H}$, exposed human $E_{H}$, infected symptomatic human $I_{1}$, infected asymptomatic human $I_{2}$, recovered Human $R$, such that the total human population, $N_{H}=S_{H}+V_{H}+E_{H}+I_{1}+I_{2}+R$. While the mosquito sub-population is divided into; susceptible mosquito $S_{M}$, exposed mosquito $E_{M}$, and infected mosquito $I_{3}$, such that the total mosquito population, $N_{M}=S_{M}+E_{M}+I_{3}$.

The parameters of the model and their values are given in Table 1, while Figure 1 is the schematic diagram of the transmission dynamics.

The susceptible human sub-population is generated at a constant rate $\Lambda_{H}$, which includes birth and immigration. The vaccinated population is generated as members of the susceptible population receive vaccination at the rate $v$, a proportion of the vaccinated with time lose their immunity at the rate $\psi$ as their vaccine wanes and move back to the susceptible population. Member of the susceptible and vaccinated populations acquire infection at the rate $\frac{\alpha_{1} b_{m} I_{M}}{N_{H}}$ and $\frac{\alpha_{1} b_{m} I_{M}(1-\varepsilon)}{N_{H}}$ respectively and move to the exposed population, where $\alpha_{1}$ is the probability of infection, $b_{m}$ biting rate of mosquito and $\varepsilon$ (where $0<\varepsilon<1$ ) is the efficacy of the imperfect vaccine. Members of the exposed population move to either symptomatic infectious population at the rate $\sigma_{1}$ or to asymptomatic infectious population at the rate $\left(1-\sigma_{1}\right)$. The recovered population is generated as both symptomatic and asymptomatic infected populations recover with lifelong immunity at the rate $\gamma$. All human population are decreased by natural death at the rate $\mu_{1}$, except the two infected populations that are decreased by disease induced death at the rate $\delta$.

The susceptible mosquito population is generated by $\Lambda_{M}$, this population is decreased by birth from infected mosquito (vertical transmission) at the rate $\beta \Lambda_{M}$; and as its members take a blood meal from either symptomatic or asymptomatic infected human (horizontal transmission) at the rate $\alpha_{2}$. The 
exposed mosquito population progresses to infected mosquito population at the rate $\sigma_{2}$. It is assumed that births from infected mosquito do not pass through the exposed class. All sub-populations of mosquito die naturally at the rate $\mu_{2}$.

Table 1. Parameters of the model Equations (1) to (9).

\begin{tabular}{|c|c|c|c|}
\hline Parameters & Meaning & Value & Reference \\
\hline$\Lambda_{H}$ & Recruitment rate of susceptible human & 0.073 & {$[19]$} \\
\hline$\alpha_{1}$ & $\begin{array}{l}\text { Contact rate of susceptible human when bitten by } \\
\text { Aides Mosquitoes }\end{array}$ & 0.24 & {$[19]$} \\
\hline$\mu_{1}$ & Natural death of human & 0.000039 & {$[20]$} \\
\hline$\sigma_{1}$ & $\begin{array}{l}\text { Progression rate of exposed human to } \\
\text { Symptomatic and Asymptomatic }\end{array}$ & 0.33 & {$[21]$} \\
\hline$\delta_{1}$ & Death rate of human due to virus infection & 0.02 & Assumed \\
\hline$\gamma$ & Recovery rate of infectious human & 0.68 & {$[15]$} \\
\hline$\Lambda_{M}$ & Birth rate of Susceptible Aides Mosquitoes & 83.75 & {$[20][22]$} \\
\hline$\beta$ & $\begin{array}{l}\text { Proportion of infectious new birth from } \\
\text { infected Aides Mosquitoes }\end{array}$ & 0.00005 & Assumed \\
\hline$\alpha_{2}$ & The rate at which susceptible Aides become infectious & 0.24 & {$[19]$} \\
\hline$\sigma_{2}$ & Progression rate of exposed Aedes & 0.285 & Assumed \\
\hline$\mu_{2}$ & Natural death rate of Aides & 0.0714 & {$[21][23]$} \\
\hline$v$ & The rate at which susceptible human receive vaccine & Variable & \\
\hline$\psi$ & The rate at which vaccine wane & Variable & \\
\hline$\varepsilon$ & Vaccine efficacy where $0<\varepsilon<1$ & Variable & \\
\hline$b_{m}$ & Biting rate of mosquito & 0.25 & [19] [24] \\
\hline
\end{tabular}

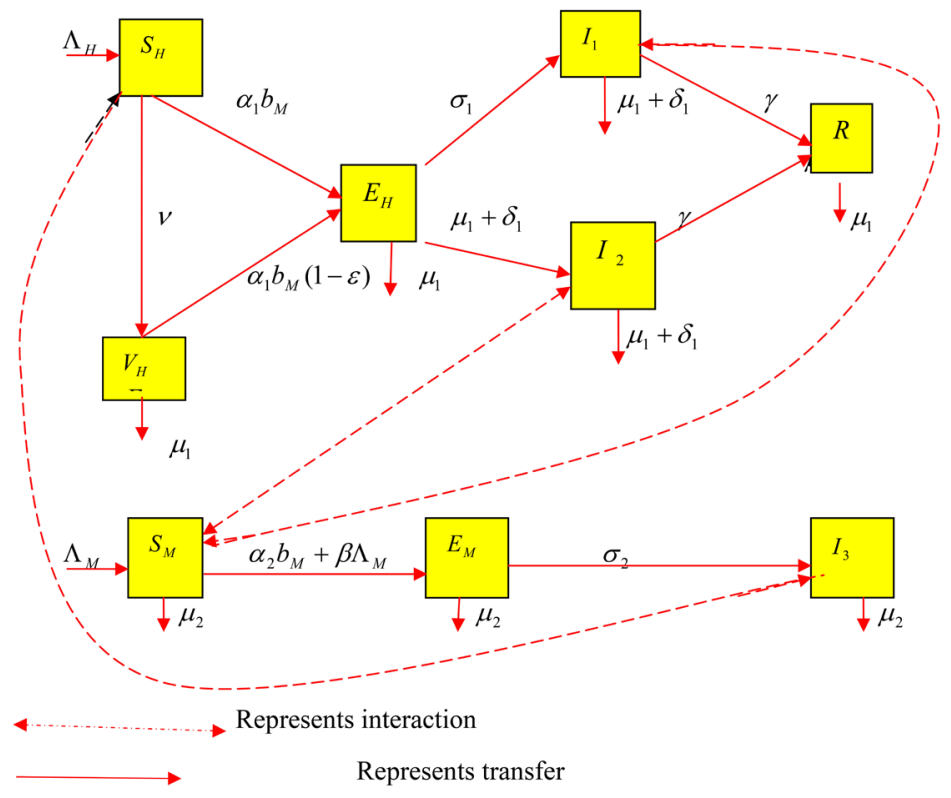

Figure 1. Schematic diagram of Chikungunya virus transmission dynamics, Equations (1) to (9). 


\subsection{The Model Equation}

From the model formulation, and schematic diagram Figure 1, we hereby present the model equations.

$$
\begin{gathered}
\frac{\mathrm{d} S_{H}}{\mathrm{~d} t}=\Lambda_{H}+\psi V_{H}-\frac{\alpha_{1} b_{M} S_{H} I_{M}}{N_{H}}-\left(v+\mu_{1}\right) S_{H}, \\
\frac{\mathrm{d} V_{H}}{\mathrm{~d} t}=v S_{H}-\frac{\alpha_{1} b_{M}(1-\varepsilon) V_{H} I_{M}}{N_{H}}-\left(\psi+\mu_{1}\right) V_{H}, \\
\frac{\mathrm{d} E_{H}}{\mathrm{~d} t}=\frac{\alpha_{1} b_{M} I_{M}}{N_{H}}\left(S_{H}+(1-\varepsilon) V_{H}\right)-\left(\sigma_{1}+\mu_{1}\right) E_{H}, \\
\frac{\mathrm{d} I_{1}}{\mathrm{~d} t}=\sigma_{1} E_{H}-\left(\gamma+\mu_{1}+\delta\right) I_{1}, \\
\frac{d I_{2}}{d t}=\left(1-\sigma_{1}\right) E_{H}-\left(\gamma+\mu_{1}+\delta\right) I_{2} \\
\frac{\mathrm{d} R}{\mathrm{~d} t}=\gamma I_{1}+\gamma I_{2}-\mu_{1} R, \\
\frac{\mathrm{d} S_{M}}{\mathrm{~d} t}=\Lambda_{M}-\frac{\alpha_{2} b_{M} S_{M}\left(I_{1}+I_{2}\right)}{N_{H}}-\beta \Lambda_{M} I_{M}-\mu_{2} S_{M}, \\
\frac{\mathrm{d} E_{M}}{\mathrm{~d} t}=\frac{\alpha_{2} S_{M} b_{M}\left(I_{1}+I_{2}\right)}{N_{H}}+\beta \Lambda_{M} I_{M}-\left(\sigma_{2}+\mu_{2}\right) E_{M}, \\
\frac{\mathrm{d} I_{M}}{\mathrm{~d} t}=\sigma_{2} E_{M}-\mu_{2} I_{M} .
\end{gathered}
$$

Adding (1) to (6) gives

$$
\frac{\mathrm{d} N_{H}}{\mathrm{~d} t}=\Lambda_{H}-\delta\left(I_{1}+I_{2}\right)-\mu_{1} N_{H} .
$$

Also adding (7) to (9), gives

$$
\frac{\mathrm{d} N_{M}}{\mathrm{~d} t}=\Lambda_{M}-\mu_{2} N_{M} .
$$

where

$$
\begin{aligned}
N_{H}(t)= & S_{H}(t)+V_{H}(t)+E_{H}(t)+I_{1}(t)+I_{2}(t)+R(t), \\
& N_{M}(t)=S_{M}(t)+E_{M}(t)+I_{M}(t) .
\end{aligned}
$$

(12) and (13) are the total human population and Aides mosquito population respectively.

\subsection{Basic Properties}

For the Chikungunya model (1) to (9) to be epidemiological meaningful, it is necessary to prove that all its state variables are non-negative for all time. This means that the solution of the model Equations (1) to (9) with non-negative initial data will remain non-negative for all time $t>0$.

Lemma 1. 
The closed set

$$
D=\left\{\begin{array}{l}
\left(S_{H}, V_{H}, E_{H}, I_{1}, I_{2}, R, S_{M}, E_{M}, I_{M}\right) \in \mathfrak{R}_{+}^{9}: \\
S_{H}, V_{H}, E_{H}, I_{1}, I_{2}, R \leq \frac{\Lambda_{H}}{\mu_{1}} ; S_{M}, E_{M}, I_{M} \leq \frac{\Lambda_{M}}{\mu_{2}} ;
\end{array}\right\} .
$$

is positively-invariant and attracting with respect to the basic model Equations (1) to (9).

\section{Proof}

From Equations (10) and (11);

$$
\frac{\mathrm{d} N_{H}}{\mathrm{~d} t} \leq \Lambda_{H}-\mu_{1} N_{H}, \frac{\mathrm{d} N_{M}}{\mathrm{~d} t} \leq \Lambda_{A}-\mu_{2} N_{M} .
$$

It follows that $\frac{\mathrm{d} N_{H}}{\mathrm{~d} t}<0$ and $\frac{\mathrm{d} N_{M}}{\mathrm{~d} t}<0$ if $N_{H}(t)>\frac{\Lambda_{H}}{\mu_{1}}$ and $N_{A}(t)>\frac{\Lambda_{M}}{\mu_{2}}$ respectively. Thus a standard comparison theorem as in Lakshmikantham and Martynyuk, [25] can be used to show that

$N_{H}(t) \leq N_{H}(0) \mathrm{e}^{-\mu_{1}(t)}+\frac{\Lambda_{M}}{\mu_{1}}\left(1-\mathrm{e}^{-\mu_{1}(t)}\right)$ and

$N_{M}(t) \leq N_{M}(0) \mathrm{e}^{-\mu_{2}(t)}+\frac{\Lambda_{M}}{\mu_{2}}\left(1-\mathrm{e}^{-\mu_{2}(t)}\right)$. In particular $N_{H}(t) \leq \frac{\Lambda_{H}}{\mu_{1}}$ and

$N_{M}(t) \leq \frac{\Lambda_{M}}{\mu_{2}}$ if $N_{H}(0) \leq \frac{\Lambda_{H}}{\mu_{1}}$ and $N_{A}(0) \leq \frac{\Lambda_{M}}{\mu_{2}}$ respectively. Thus $D$ is posi-

tively-invariant. Further, if $N_{H}(0)>\frac{\Lambda_{H}}{\mu_{1}}$, and $N_{M}(0)>\frac{\Lambda_{M}}{\mu_{2}}$, then either the solution enters $D$ in finite time or $N_{H}(t)$ approaches $\frac{\Lambda_{H}}{\mu_{1}}$, and $N_{M}(t)$ approaches $\frac{\Lambda_{M}}{\mu_{2}}$, and the infected variables $E_{H}, I_{1}, I_{2}, E_{A}, I_{3}$ approaches 0.

Hence $D$ is attracting, that is all solutions in $\mathfrak{R}_{+}^{9}$ eventually enters $D$. Thus in $D$, the basic model Equations (1) to (9) is well posed epidemiologically and mathematically according to [26]. Hence it is sufficient to study the dynamics of the basic model Equations (1) to (9).

Lemma 2. Let the initial data $F(0) \geq 0$, where

$$
F(t)=\left(S_{H}, V_{H}, E_{H}, I_{1}, I_{2}, R, S_{M}, E_{M}, I_{M}\right) .
$$

Then the solution $F(t)$ of the Chikungunya virus model (1) to (9) are non-negative for all $t \geq 0$. Furthermore form (10) and (11),

$$
\limsup _{t \rightarrow \infty} N_{H}(t)=\frac{\Lambda_{H}}{\mu_{1}+\delta} \text { and } \limsup _{t \rightarrow \infty} N_{M}(t)=\frac{\Lambda_{M}}{\mu_{2}} .
$$

\section{Proof}

$t_{1}=\sup \{t>0: F(t)>0 \in[0, t]\}$. Thus $t_{1}>0$. It follows from (1) that 


$$
\begin{aligned}
& \frac{\mathrm{d}}{\mathrm{d} t}\left\{S_{H}(t) \exp \left[\alpha_{1} b_{m} \int_{0}^{t_{1}} \frac{I_{M}}{N_{H}}(\xi) \mathrm{d} \xi+\left(v+\mu_{1}\right) t\right]\right\} \\
& =\left(\Lambda_{H}+\psi V_{H}\right) \exp \left[\alpha_{1} b_{M} \int_{0}^{t_{1}} \frac{I_{M}}{N_{H}}(\xi) \mathrm{d} \xi+\left(v+\mu_{1}\right) t\right]
\end{aligned}
$$

So that,

$$
\begin{aligned}
& \frac{\mathrm{d}}{\mathrm{d} t} S_{H}\left(t_{1}\right) \exp \left[\alpha_{1} b_{m} \int_{0}^{t_{1}} \frac{I_{M}}{N_{H}}(\xi) \mathrm{d} \xi+\left(v+\mu_{1}\right) t_{1}\right]-S_{H}(0) \\
& =\int_{0}^{t_{1}}\left(\Lambda_{H}+\psi V_{H}\right) \exp \left[\alpha_{1} b_{m} \int_{0}^{P} \frac{I_{M}}{N_{H}}(\xi) \mathrm{d} \xi+\left(v+\mu_{1}\right) p\right] \mathrm{d} p
\end{aligned}
$$

Hence,

$$
\begin{aligned}
S_{H}\left(t_{1}\right)= & S_{H}(0) \exp \left[-\alpha_{1} b_{m} \int_{0}^{t_{1}} \frac{I_{M}}{N_{H}}(\xi) \mathrm{d} \xi+\left(v+\mu_{1}\right) t_{1}\right] \\
& +\exp \left[-\alpha_{1} b_{m} \int_{0}^{t_{1}} \frac{I_{M}}{N_{H}}(\xi) \mathrm{d} \xi+\left(v+\mu_{1}\right) t_{1}\right] \\
& \int_{0}^{t_{1}}\left(\Lambda_{H}+\psi V_{H}\right) \exp \left[\alpha_{1} b_{M} \int_{0}^{P} \frac{I_{M}}{N_{H}}(\xi) \mathrm{d} \xi+\left(v+\mu_{1}\right) p\right] \mathrm{d} p>0 .
\end{aligned}
$$

Similarly, it can be shown that $F>0$, for all $t>0$.

For the second part of the proof, note that,

$$
\begin{aligned}
& 0<V_{H}(t) \leq N_{H}(t), 0<E_{H}(t) \leq N_{H}(t), 0<I_{1}(t) \leq N_{H}(t), \\
& 0<I_{2}(t) \leq N_{H}(t), 0<R(t) \leq N_{H}(t), 0<S_{M}(t) \leq N_{M}(t), \\
& 0<E_{M}(t) \leq N_{M}(t), 0<I_{M}(t) \leq N_{M}(t)
\end{aligned}
$$

From Equations (10) and (11),

$$
\frac{\Lambda_{H}}{\mu_{1}+\delta} \leq \liminf _{t \rightarrow \infty} N_{H}(t) \leq \limsup _{t \rightarrow \infty} N_{H}(t)=\frac{\Lambda_{H}}{\mu_{1}+\delta},
$$

and

$$
\frac{\Lambda_{M}}{\mu_{2}} \leq \liminf _{t \rightarrow \infty} N_{M}(t) \leq \limsup _{t \rightarrow \infty} N_{M}(t)=\frac{\Lambda_{M}}{\mu_{2}} .
$$

as required.

\section{Results}

\subsection{Local Stability of Disease Free Equilibrium (DFE)}

The basic model (1) to (9) has a DFE, $E_{0}$ obtained by setting the right-hand sides of the model equations to zero, which gives:

$$
\begin{aligned}
E_{0} & =\left(S_{H}^{*}, V_{H}^{*}, E_{H}^{*}, I_{1}^{*}, I_{2}^{*}, S_{M}^{*}, E_{M}^{*}, I_{M}^{*}\right) \\
& =\left(\frac{\Lambda_{H}\left(\psi+\mu_{1}\right)}{\left(\psi+\mu_{1}+v\right) \mu_{1}}, \frac{v \Lambda_{H}}{\left(\psi+\mu_{1}+v\right) \mu_{1}}, 0,0,0,0, \frac{\Lambda_{M}}{\mu_{2}}, 0,0\right)
\end{aligned}
$$

The linear stability of $E_{0}$ can be established using the next generation Matrix operator method on the system (I) to (9). Using the notation in [23], the matric- 
es $F$ and $V$, for the new infection terms and the remaining transfer terms, are, respectively, given by,

$$
F=\left[\begin{array}{ccccc}
0 & 0 & 0 & 0 & \frac{\alpha_{1} b_{m}\left[S_{H}^{*}+(1-\varepsilon) V_{H}^{*}\right]}{N_{H}^{*}} \\
0 & 0 & 0 & 0 & 0 \\
0 & 0 & 0 & 0 & 0 \\
0 & \frac{\alpha_{2} b_{m} S_{M}^{*}}{N_{H}^{*}} & \frac{\alpha_{2} b_{m} S_{M}^{*}}{N_{H}^{*}} & 0 & 0 \\
0 & 0 & 0 & 0 & 0
\end{array}\right]
$$

and,

$$
V=\left[\begin{array}{ccccc}
K_{3} & 0 & 0 & 0 & 0 \\
-\sigma_{1} & K_{4} & 0 & 0 & 0 \\
-\left(1-\sigma_{1}\right) & 0 & K_{4} & 0 & 0 \\
0 & 0 & 0 & K_{5} & 0 \\
0 & 0 & 0 & -\sigma_{2} & K_{6}
\end{array}\right]
$$

where,

$$
\begin{gathered}
K_{1}=v_{1}+\mu_{1}, K_{2}=\psi+\mu_{1}, K_{3}=\sigma_{1}+\mu, \\
K_{4}=\gamma+\mu_{1}+\delta, K_{5}=\sigma_{2}+\mu_{2}, K_{6}=\mu_{1}-\beta \Lambda_{M} \\
R_{c}=\frac{1}{2}\left(\frac{M_{1}+\sqrt{M_{2}+M_{3}}}{M_{4}}\right), \\
M_{1}=\Lambda_{M} \beta K_{3} K_{4} K_{5} N_{H}^{*}, \\
M_{2}=\Lambda_{M} \beta K_{3} K_{4} K_{5} N_{H}^{*}, \\
M_{3}=4 K_{3} K_{4} K_{5} K_{6} \alpha_{2} \sigma_{2} b_{m} S_{M}^{*}\left(\alpha_{1} b_{m} S_{H}^{*}+(1-\varepsilon)\right) N_{H}^{*}, \\
M_{4}=\beta K_{3} K_{4} K_{5} K_{6} N_{H}^{*} .
\end{gathered}
$$

Hence using theorem 2 of [23] the following results are established.

Theorem 1 The disease free equilibrium, $E_{0}$ of the model (2.1) to (2.9) is locally asymptotically stable $(L A S)$ if $R_{c}<1$, and unstable if $R_{c}>1$.

\subsection{Global Stability of Disease Free Equilibrium}

Consider the feasible region:

$$
\begin{gathered}
D_{1}=\left\{X \in D_{1}: S_{H} \leq S_{H}^{*}, V_{H} \leq V_{H}^{*}, R \leq R^{*}, S_{M} \leq S_{M}^{*}\right\}, \\
X=\left\{S_{H}, V_{H}, E_{H}, I_{1}, I_{2}, R, S_{M}, E_{M}, I_{M}\right\} .
\end{gathered}
$$

Lemma 3. The region $D_{1}$ is positively invariant for the Chikungunya model 
Equations (1) to (9).

\section{Proof}

From Equations (1) to (9) and (20),

we have that, the only non-zero compartments at disease free equilibrium are;

$$
\begin{aligned}
& \frac{d S_{H}}{d t}=\Lambda_{H}+\psi V_{H}-\frac{\alpha_{1} b_{M} S_{H} I_{M}}{N_{H}}-\left(v+\mu_{1}\right) S, \\
& \frac{d V_{H}}{d t}=v S_{H}-\frac{\alpha_{1} b_{M}(1-\varepsilon) V_{H} I_{M}}{N_{H}}-\left(\psi+\mu_{1}\right) V_{H}, \\
& \frac{d S_{M}}{d t}=\Lambda_{M}-\frac{\alpha_{2} b_{M} S_{M}\left(I_{1}+I_{2}\right)}{N_{H}}-\beta \Lambda_{M} I_{M}-\mu_{2} S_{M}
\end{aligned}
$$

Such that,

$$
\begin{aligned}
\frac{\mathrm{d} S_{H}}{\mathrm{~d} t} & =\Lambda_{H}+\psi V_{H}-\frac{\alpha_{1} b_{m} S_{H} I_{M}}{N_{H}}-\left(v+\mu_{1}\right) S_{H}, \\
& \leq \Lambda_{H}+\psi V_{H}-\left(v+\mu_{1}\right) S_{H} \\
& \leq\left(v+\mu_{1}\right)\left[\frac{\Lambda_{H}\left(\psi+\mu_{1}\right)}{\left(\psi+\mu_{1}+v\right) \mu_{1}}+\psi \frac{v \Lambda_{H}}{\left(\psi+\mu_{1}+v\right) \mu_{1}}-S_{H}\right], \\
& =\left(v+\mu_{1}\right)\left(S_{H}^{*}+\psi V_{H}^{*}-S_{H}\right)
\end{aligned}
$$

Hence,

$$
S_{H}(t) \leq S_{H}^{*}+\psi V_{H}^{*}-\left[S_{H}^{*}-\psi V_{H}^{*}-S_{H}(0)\right] \mathrm{e}^{-\left(v+\mu_{1}\right) t}
$$

Thus if $N_{H}^{*}=\frac{\Lambda_{H}}{\mu_{1}} \quad$ and $\quad S_{H}(0) \leq S_{H}^{*}+\psi V_{H}^{*} \quad$ for $\quad$ all $t \geq 0$, then $S_{H}(t) \leq S_{H}^{*}+\psi V_{H}^{*}$ for all $t \geq 0$.

Similarly, it follows from Equation (7) of our model and (20) where $S_{M}^{*}=\frac{\Lambda_{M}}{\mu_{2}}$.

We have that,

$$
\begin{aligned}
\frac{\mathrm{d} S_{M}}{\mathrm{~d} t} & =\Lambda_{M}-\frac{\alpha_{2} b_{M} S_{M}\left(I_{1}+I_{2}\right)}{N_{H}}-\beta \Lambda_{M} I_{M}-\mu_{2} S_{M} \\
& \leq \Lambda_{M}-\mu_{2} S_{M} \leq \mu_{2}\left[\frac{\Lambda_{M}}{\mu_{2}}-S_{M}\right]=\mu_{2}\left(S_{M}^{*}-S_{M}\right)
\end{aligned}
$$

Hence,

$$
S_{M}(t) \leq S_{M}^{*}-\left[S_{M}^{*}-S_{M}(0)\right] \mathrm{e}^{-\mu_{2} t}
$$

Thus if $N_{M}^{*}=\frac{\Lambda_{M}}{\mu_{2}}$ and $S_{M}(0) \leq S_{M}^{*}$ for all $t \geq 0$, then $S_{M}(t) \leq S_{M}^{*}$ for all $t \geq 0$.

In summary, we have shown that $D_{1}$ is positively invariant and attracting with respect to the solutions of our model Equations (1) to (9).

Theorem 2

The DFE of the basic model (1) to (9) is Global Asymptotical Stability (GAS) in $D_{1}$, whenever $R_{C} \leq 1$. 


\section{Proof}

To prove the GAS of the DFE we adopt the approach in [27].

Let $X=\left(S_{H}, V_{H}, R, S_{M}\right)$ and $Z=\left(E_{H}, I_{1}, I_{2}, E_{M}, I_{M}\right)$ and group our model Equations (1) to (8) into:

$$
\begin{aligned}
& \frac{\mathrm{d} X}{\mathrm{~d} t}=F(X, 0), \\
& \frac{\mathrm{d} Z}{\mathrm{~d} t}=G(X, Z) .
\end{aligned}
$$

where $F(X, 0)$ is the right hand side of $\dot{S}_{H}, \dot{V}_{H}, \dot{R}, \dot{S}_{M}$ with $E_{H}=I_{1}=I_{2}=E_{M}=I_{M}=0$ and $G(X, Z)$, the right hand side of $\dot{E}_{H}, \dot{I}_{1}, \dot{I}_{2}, \dot{E}_{M}, \dot{I}_{M}$. Next we consider the reduced system:

$$
\begin{aligned}
\frac{\mathrm{d} X}{\mathrm{~d} t} & =F(X, 0) \text { given as, } \\
\frac{\mathrm{d} S_{H}}{\mathrm{~d} t} & =\Lambda_{H}-\mu_{1} S_{H}, \\
\frac{\mathrm{d} V_{H}}{\mathrm{~d} t} & =v S_{H}-\left(\psi+\mu_{1}\right) V_{H}, \\
\frac{\mathrm{d} R}{\mathrm{~d} t} & =-\mu_{1} R, \\
\frac{\mathrm{d} S_{M}}{\mathrm{~d} t} & =\Lambda_{M}-\mu_{2} S_{M} . \\
\text { Let } X^{*}=\left(S_{H}^{*}, V_{H}^{*}, R^{*}, S_{M}^{*}\right) & =\left(\frac{\Lambda_{H}\left(\psi+\mu_{1}\right)}{\left(\psi+\mu_{1}+v\right) \mu_{1}}, \frac{v \Lambda_{H}}{\left(\psi+\mu_{1}+v\right) \mu_{1}}, 0, \frac{\Lambda_{M}}{\mu_{2}}\right)
\end{aligned}
$$

be an equilibrium of (37) we show that $X^{*}$ is a global stable equilibrium in $D_{1}$.

To do this, we solve the Equations (37), which gives

$$
\begin{aligned}
S_{H}(t)= & \left(\frac{\Lambda_{H}\left(\psi+\mu_{1}\right)}{\left(\psi+\mu_{1}+v\right) \mu_{1}}+\psi V_{H}^{*}\right)-\left(\frac{\Lambda_{H}\left(\psi+\mu_{1}\right)}{\left(\psi+\mu_{1}+v\right) \mu_{1}}+\psi V_{H}^{*}\right) \mathrm{e}^{-\left(\left(\psi+\mu_{1}+v\right) \mu_{1}\right) t} \\
& +S_{H}(0) \mathrm{e}^{\left(\left(\psi+\mu_{1}+v\right) \mu_{1}\right) t} \\
S_{H}(t) \rightarrow & \frac{\Lambda_{H}\left(\psi+\mu_{1}\right)}{\left(\psi+\mu_{1}+v\right) \mu_{1}}+\psi V_{H}^{*},
\end{aligned}
$$

as $t \rightarrow \infty$.

$$
\begin{aligned}
& V_{H}(t)=\frac{v \Lambda_{H}}{\left(\psi+\mu_{1}+v\right) \mu_{1}}-\frac{v \Lambda_{H}}{\left(\psi+\mu_{1}+v\right) \mu_{1}} \mathrm{e}^{-\left(\left(\psi+\mu_{1}+v\right) \mu_{1}\right) t}+V_{H}(0) \mathrm{e}^{\left(\left(\psi+\mu_{1}+v\right) \mu_{1}\right) t}, \\
& V_{H}(t) \rightarrow \frac{v \Lambda_{H}}{\left(\psi+\mu_{1}+v\right) \mu_{1}},
\end{aligned}
$$

as $t \rightarrow \infty$.

$$
R(t)=R(0) \mathrm{e}^{-\mu_{1} t}, R(t) \rightarrow 0
$$

as $t \rightarrow \infty$.

$$
S_{M}(t)=\frac{\Lambda_{M}}{\mu_{2}}-\frac{\Lambda_{M}}{\mu_{2}} \mathrm{e}^{-\mu_{2} t}+S_{M}(0) \mathrm{e}^{-\mu_{2} t}, S_{M}(t) \rightarrow \frac{\Lambda_{M}}{\mu_{2}},
$$


as $t \rightarrow \infty$.

This asymptotic dynamics is independent of initial conditions in $D$. Hence the solution of xxx converges globally in $D_{1}$.

Next we are required to show that $G(X, Z)$ satisfies the following two conditions in [19] pp246 namely;

$$
\begin{aligned}
& G(X, 0)=0, \\
& G(X, Z)=D_{Z} \hat{G}\left(X^{*}, 0\right) Z-\hat{G}(X, Z), \hat{G}(X, Z) \geq 0,
\end{aligned}
$$

where,

$$
\left(X^{*}, 0\right)=\left(\frac{\Lambda_{H}\left(\psi+\mu_{1}\right)}{\left(\psi+\mu_{1}+v\right) \mu_{1}}, \frac{v \Lambda_{H}}{\left(\psi+\mu_{1}+v\right) \mu_{1}}, 0, \frac{\Lambda_{M}}{\mu_{2}}\right) .
$$

and $D_{Z} G\left(X^{*}, 0\right)$ is the Jacobian of $G(X, Z)$ taken with respect to $\left(E_{H}, I_{1}, I_{2}, E_{M}, I_{M}\right)$ and evaluated at $\left(X^{*}, 0\right)$, which is an M-Matrix (the off diagonal elements are non-negative).

Thus,

$$
\begin{aligned}
& D_{Z} G\left(X^{*}, 0\right)=\left(\begin{array}{ccccc}
-k_{3} & 0 & 0 & 0 & Q_{1} \\
\sigma_{1} & -k_{4} & 0 & 0 & 0 \\
1-\sigma_{1} & 0 & -k_{4} & 0 & 0 \\
0 & \frac{\alpha_{2} b_{m} S_{M}^{*}}{N_{H}^{*}} & \frac{\alpha_{2} b_{m} S_{M}^{*}}{N_{H}^{*}} & -k_{5} & 0 \\
0 & 0 & 0 & \sigma_{2} & -k_{6}
\end{array}\right) \\
& \hat{G}(X, Z)=\left(\begin{array}{ccccc}
0 & 0 & 0 & 0 & Q_{2} I_{M} \\
0 & 0 & 0 & 0 & 0 \\
0 & 0 & 0 & 0 & 0 \\
0 & \alpha_{2} b_{M} \frac{N_{H}^{*}}{S_{M}^{*}} Q_{3} & \alpha_{2} b_{M} \frac{N_{H}^{*}}{S_{M}^{*}} Q_{3} & 0 & 0 \\
0 & 0 & 0 & 0 & \frac{1}{\beta \Lambda_{M}}
\end{array}\right),
\end{aligned}
$$

where,

$$
\begin{aligned}
Q_{1} & =\frac{\alpha_{1} b_{m} S_{H}^{*}+(1-\varepsilon)+V_{H}^{*}}{N_{H}^{*}}, \\
Q_{2} & =\frac{N_{H}^{*}}{S_{H}^{*}+(1-\varepsilon)+V_{H}^{*}}\left(1-\frac{N_{H}^{*}}{S_{H}^{*}+(1-\varepsilon)+V_{H}^{*}} \frac{S_{H}+(1-\varepsilon) V_{H}}{N_{H}}\right), \\
Q_{3} & =\left(1-\frac{N_{H}^{*}}{S_{M}^{*}} \frac{S_{M}}{N_{H}} I_{1}\right) \\
Q_{4} & =\left(1-\frac{N_{H}^{*}}{S_{M}^{*}} \frac{S_{M}}{N_{H}} I_{2}\right) .
\end{aligned}
$$

Further $S_{H} \leq S_{H}^{*}, V_{H} \leq V_{H}^{*}$ and $S_{M} \leq S_{M}^{*}$ in $D_{1}$. Thus, it follows that 
$\left(1-\frac{S_{H}}{S_{H}^{*}}\right)>0,\left(1-\frac{V_{H}}{V_{H}^{*}}\right)>0$ and $\left(1-\frac{S_{M}}{S_{M}^{*}}\right)>0$. Hence $\hat{G}(X, Z) \geq 0$.

Therefore, by the theorem 2 in [28], the disease-free equilibrium is globally asymptotically stable since in the absence of disease induced mortality the human population is constant.

\subsection{Sensitivity Analysis}

Here we present the sensitivity index of the parameters of the effective reproductive number $\left(R_{C}\right)$. Sensitivity tells us how important each parameter is to disease transmission. Such information, is crucial not only to experimental design, but also to data assimilation and reduction of complex nonlinear model [29]. Sensitivity Analysis is commonly used to determine the robustness of model prediction to parameter values, since there are usually errors in data collection and presumed parameter values. It is used to determine parameters that have high impact on the $\left(R_{C}\right)$ and should be targeted by intervention strategies. Sensitivity indexes allows us to measure the relative changes in a variable when a parameter changes. The normalized forward sensitivity index of a variable with respect to a parameter is the ratio of relative changes in the parameter when the variable is a differentiable function of the parameter. The sensitivity index may be alternatively defined using partial derivatives. The sensitivity index of our model is given in Table 2.

Table 2. Sensitivity analysis index for the effective basic reproductive number.

\begin{tabular}{|c|c|}
\hline Parameter & Sensitivity index \\
\hline$\alpha_{1}$ & 0.06 \\
\hline$\alpha_{2}$ & 0.37 \\
\hline$\mu_{1}$ & 0.63 \\
\hline$\mu_{2}$ & 0.87 \\
\hline$\sigma_{1}$ & 0.02 \\
\hline$\sigma_{2}$ & 0.87 \\
\hline$\beta$ & 1.25 \\
\hline$\psi$ & -0.12 \\
\hline$\varepsilon$ & -0.86 \\
\hline$\delta$ & 0.13 \\
\hline$\gamma$ & 0.5 \\
\hline$\Lambda_{H}$ & 0.62 \\
\hline$\Lambda_{M}$ & 1.6 \\
\hline$v$ & 0.46 \\
\hline
\end{tabular}


From Table 2, the most sensitive parameter of $R_{C}$ is the recruitment rate of susceptible mosquito $\left(\Lambda_{M}\right)$ followed by the proportion of infectious new birth from infected mosquito $(\beta)$ while the natural birth rate of mosquito $\left(\mu_{2}\right)$ and the rate at which exposed mosquito become infectious $\left(\sigma_{2}\right)$ are equally sensitive to the $R_{C}$ according to the model. This means that any policy or practice capable of reducing these parameters will go a long way in reducing the menace of Chikungunya and at the long run, result to eradication.

\section{Endemic Equilibrium}

$$
\text { Let } E_{1}=\left(S_{H}^{* *}, V_{H}^{* *}, E_{H}^{* *}, R^{* *}, I_{1}^{* *}, I_{2}^{* *}, R^{* *}, S_{M}^{* *}, E_{M}^{* *}, I_{M}^{* *}\right) \text {, }
$$

represents any arbitrary endemic equilibrium of the model (1) to (9). Further, let

$$
\lambda_{H}^{* *}=\frac{\alpha_{1} b_{m} I_{M}^{* *}}{N_{H}^{* *}}, \lambda_{M}^{* *}=\frac{\alpha_{2} b_{M}\left(I_{1}^{* *}+I_{2}^{* *}\right)}{N_{H}^{* *}} .
$$

be the forces of infection of humans and vectors at steady state, respectively. Solving (1) to (9) in terms of $\lambda_{H}^{* *}$ and $\lambda_{M}^{* *}$, we have;

$$
\begin{aligned}
& S_{H}^{* *}=\frac{\Lambda_{H}\left(\lambda_{H}^{* *}+k_{2}\right)}{\left(\lambda_{H}^{* *}+k_{2}\right)\left(\lambda_{H}^{* *}+k_{1}\right)+\psi v}, V_{H}^{* *}=\frac{v \Lambda_{H}}{\left(\lambda_{H}^{* *}+k_{2}\right)\left(\lambda_{H}^{* *}+k_{1}\right)+\psi v}, \\
& E_{H}^{* *}=\frac{\Lambda_{H} \lambda_{H}^{* *}\left(\lambda_{H}^{* *}+k_{2}\right)+(1-\varepsilon)}{\left(\left(\lambda_{H}^{* *}+k_{2}\right)\left(\lambda_{H}^{* *}+k_{1}\right)-\psi v\right) k_{3}}, I_{1}^{* *}=\frac{\sigma_{1} \Lambda_{H} \lambda_{H}^{* *}\left(\left(\lambda_{H}^{* *}+k_{2}\right)+(1-\varepsilon)\right) v}{\left(\left(\lambda_{H}^{* *}+k_{2}\right)\left(\lambda_{H}^{* *}+k_{1}\right)-\psi v\right) k_{3} k_{4}}, \\
& I_{2}^{* *}=\frac{\left(1-\sigma_{1}\right) \Lambda_{H} \lambda_{H}^{* *}\left(\left(\lambda_{H}^{* *}+k_{2}\right)+(1-\varepsilon)\right) v}{\left(\left(\lambda_{H}^{* *}+k_{2}\right)\left(\lambda_{H}^{* *}+k_{1}\right)-\psi v\right) k_{3} k_{4}}, R^{* *}=\frac{\gamma \Lambda_{H} \lambda_{H}^{* *}\left(\left(\lambda_{H}^{* *}+k_{2}\right)+(1-\varepsilon)\right) v}{\left(\left(\lambda_{H}^{* *}+k_{2}\right)\left(\lambda_{H}^{* *}+k_{1}\right)-\psi v\right) k_{3} k_{4} \mu_{1}}, \\
& S_{M}^{* *}=\frac{k_{5} k_{6} \Lambda_{M}}{\lambda_{M}^{* *}\left(k_{5} k_{6}+\beta \Lambda_{M} \sigma_{2}\right)+\mu_{2} k_{5} k_{6}}, E_{M}^{* *}=\frac{k_{6} \Lambda_{M} \lambda_{M}^{* *}}{\lambda_{M}^{* *}\left(k_{5} k_{6}+\beta \Lambda_{M} \sigma_{2}\right)+\mu_{2} k_{5} k_{6}}, \\
& I_{M}^{* * *}=\frac{\sigma_{2} \Lambda_{M} \lambda_{M}^{* *}}{\lambda_{M}^{* *}\left(k_{5} k_{6}+\beta \Lambda_{M} \sigma_{2}\right)+\mu_{2} k_{5} k_{6}} .
\end{aligned}
$$

Substituting (20) into (19) we have;

$$
\begin{aligned}
& \lambda_{M}^{* *}=\frac{\alpha_{2} b_{m} \Lambda_{M} \lambda_{H}^{* *}\left(\left(\lambda_{H}^{* *}+k_{2}\right)+(1-\varepsilon)\right) v}{\left(\left(\lambda_{H}^{* *}+k_{2}\right)\left(\lambda_{H}^{* *}+k_{1}\right)-\psi v\right) k_{3} k_{4}}, \\
& \lambda_{H}^{* *}=A\left(\lambda_{H}^{* *}\right)^{4}+B\left(\lambda_{H}^{* *}\right)^{3}+C\left(\lambda_{H}^{* *}\right)^{2}+D\left(\lambda_{H}^{* *}\right)-E .
\end{aligned}
$$

where,

$$
\begin{aligned}
A= & \left(\alpha_{2} b_{m} \Lambda_{H} k_{5} k_{6}+\beta \Lambda_{M} \sigma_{2}\right)\left(\mu_{1}+k_{4} \mu_{1}+\gamma\right) \\
B=\left(T_{2} k_{5} k_{6}\right. & \left.+\beta \Lambda_{M} \sigma_{2}\right)\left(k_{3} k_{4}\left(\mu_{1} k_{2}+\psi\right)\right)-\left(\alpha_{1} \alpha_{2}\left(b_{m}\right)^{2} \Lambda_{H} \sigma_{2}\right) k_{3} k_{4} \mu_{1}, \\
C= & {\left[\alpha_{2} b_{m} \Lambda_{H} \sigma_{2}\left(k_{2}+(1-\varepsilon) v\right) k_{5} k_{6}\right.} \\
& \left.+\beta \Lambda_{M} \sigma_{2}\left(k_{3} k_{4} \mu_{1} k_{2}+k_{1} \mu_{1}+k_{4} \mu_{1}+\gamma(1-\varepsilon) v\right)\right] \\
& -\alpha_{1} \alpha_{2}\left(b_{m}\right)^{2} \Lambda_{H} \sigma_{2} k_{3} k_{4} \mu_{1}\left(k_{1}+k_{2}\right) \\
& +\alpha_{1} \alpha_{2}\left(b_{m}\right)^{2} \Lambda_{H} \sigma_{2}\left(k_{2}+(1-\varepsilon) v\right) k_{3} k_{4} \mu_{1}
\end{aligned}
$$




$$
\begin{aligned}
D= & \left(\alpha_{2} b_{m} \Lambda_{H} \sigma_{2}\left(k_{2}+(1-\varepsilon) v\right)\left(\alpha_{2} b_{m} \Lambda_{H} k_{5} k_{6}+\beta \Lambda_{M} \sigma_{2}\right)\left(k_{3} k_{4}\left(\mu_{1} k_{2}+v\right)\right)\right) \\
& -\left(\alpha_{1} \alpha_{2}\left(b_{m}\right)^{2} \Lambda_{H} \sigma_{2}\left(k_{2}+(1-\varepsilon) v\right)\left(k_{1}+k_{2}\right) k_{3} k_{4} \mu_{1}\right) \\
& +\left(k_{1} k_{2} k_{3} k_{4}-\psi v k_{3} k_{4} \mu_{1}\right)\left(\alpha_{1} \alpha_{2}\left(b_{m}\right)^{2} \Lambda_{H} \sigma_{2}\right) \\
& E=\alpha_{1} \alpha_{2}\left(b_{m}\right)^{2} \Lambda_{H} \sigma_{2}\left(k_{2}+(1-\varepsilon) v\right) k_{1} k_{2} k_{3} k_{4}-\psi v k_{3} k_{4} \mu_{1}
\end{aligned}
$$

Theorem 3.6. The Chikungunya basic model (1) to (9) undergoes backward bifurcation whenever the coefficient a in equation is positive.

Proof. To prove this theorem, we use the Centre Manifold theory as in Castillo-Chavez and songs [30] [31] see the theorem in Appendix A.

Let $S_{H}=x_{1}, V_{H}=x_{2}, E_{H}=x_{3}, I_{1}=x_{4}, I_{2}=x_{5}, R=x_{6}, \quad S_{M}=x_{7}$, $E_{M}=x_{8} \quad, \quad I_{M}=x_{9} \quad$ so that $\quad N_{H}=x_{1}+x_{2}+x_{3}+x_{4}+x_{5}+x_{6} \quad$ and $N_{M}=x_{7}+x_{8}+x_{9}$. Further by using vector notation $X=\left(x_{1}+x_{2}+x_{3}+x_{4}+x_{5}+x_{6}+x_{7}+x_{8}+x_{9}\right)^{\mathrm{T}}$ Equations (1) to (9) can be written as $\frac{\mathrm{d} X}{\mathrm{~d} t}=\left(f_{1}+f_{2}+f_{3}+f_{4}+f_{5}+f_{6}+f_{7}+f_{8}+f_{9}\right)^{\mathrm{T}}$ as follow:

$$
\left.\begin{array}{rl}
\frac{\mathrm{d} x_{1}}{\mathrm{~d} t} & =\Lambda_{H}+\psi V_{H}-\frac{\alpha_{1} b_{M} x_{1} x_{9}}{x_{1}+x_{2}+x_{3}+x_{4}+x_{5}+x_{6}}-k x_{1}, \\
\frac{\mathrm{d} x_{2}}{\mathrm{~d} t} & =v x_{2}-\frac{\alpha_{1} b_{M}(1-\varepsilon) x_{2} x_{9}}{x_{1}+x_{2}+x_{3}+x_{4}+x_{5}+x_{6}}-k_{2} x_{2}, \\
\frac{\mathrm{d} x_{3}}{\mathrm{~d} t} & =\frac{\alpha_{1} b_{M} x_{9}}{x_{1}+x_{2}+x_{3}+x_{4}+x_{5}+x_{6}}\left(x_{1}+(1-\varepsilon) x_{2}\right)-k_{3} x_{3}, \\
\frac{\mathrm{d} x_{4}}{\mathrm{~d} t} & =\sigma_{1} x_{3}-k_{4} x_{4}, \\
\frac{\mathrm{d} x_{5}}{\mathrm{~d} t} & =\left(1-\sigma_{1}\right) x_{3}-\left(\gamma+\mu_{1}+\delta\right) x_{5}, \\
\frac{\mathrm{d} x_{6}}{\mathrm{~d} t} & =\gamma x_{4}+\gamma x_{5}-\mu_{1} x_{6}, \\
\frac{\mathrm{d} x_{7}}{\mathrm{~d} t} & =\Lambda_{M}-\frac{\alpha_{2} b_{M} x_{7}\left(x_{4}+x_{5}\right)}{x_{1}+x_{2}+x_{3}+x_{4}+x_{5}+x_{6}}-\beta \Lambda_{M} x_{9}-\mu_{2} x_{7}, \\
\frac{\mathrm{d} x_{8}}{\mathrm{~d} t} & =\frac{\alpha_{2} S_{M} b_{M}\left(x_{4}+x_{5}\right)}{x_{1}+x_{2}+x_{3}+x_{4}+x_{5}+x_{6}}+\beta \Lambda_{M} x_{9}-k_{5} x_{8}, \\
\frac{\mathrm{d} x_{9}}{\mathrm{~d} t} & =\sigma_{2} x_{8}-k_{6} x_{9} .
\end{array}\right\}
$$

Because it's not always convenient to use $R_{C}=1$ as bifurcation parameter, we choose $P=P^{*}$ where $P^{*}=\alpha_{2} b_{m}$ as the bifurcation parameter such that,

$$
P^{*}=\frac{1}{2}\left(\frac{M_{4}}{M_{1}+\sqrt{M_{2}+M_{5}}}\right),
$$

where

$$
M_{5}=4 k_{3} k_{4} k_{5} k_{6} \alpha_{2} x_{1}^{*}\left(\alpha_{7} b_{m} x_{1}^{*}+(1-\varepsilon) x_{2}^{*}\right)
$$


The Jacobian of (57) evaluated at $E_{0}$ with $\alpha_{2} b_{m}=P^{*}$, denoted by $J^{*}$ is given

$$
J^{*}=\left[\begin{array}{ccccccccc}
-k_{1} & 0 & 0 & 0 & 0 & 0 & 0 & 0 & -Q_{5} \\
v & -k_{2} & 0 & 0 & 0 & 0 & 0 & 0 & -Q_{6} \\
0 & 0 & -k_{3} & 0 & 0 & 0 & 0 & 0 & 0 \\
0 & 0 & \sigma_{1} & -k_{4} & 0 & 0 & 0 & 0 & 0 \\
0 & 0 & 1-\sigma_{1} & 0 & -k_{4} & 0 & 0 & 0 & 0 \\
0 & 0 & 0 & \gamma & \gamma & -\mu_{1} & 0 & 0 & 0 \\
0 & 0 & 0 & Q_{7} & Q_{7} & 0 & \mu_{2} & 0 & -\beta \Lambda_{M} \\
0 & 0 & 0 & Q_{7} & Q_{7} & 0 & 0 & -k_{5} & 0 \\
0 & 0 & 0 & 0 & 0 & 0 & 0 & \sigma_{2} & -k_{6}
\end{array}\right]
$$

where,

$$
\begin{aligned}
& Q_{5}=\frac{\alpha_{1} b_{m} x_{1}^{*}}{x_{1}+x_{2}+x_{3}+x_{4}+x_{5}+x_{6}}, \\
& Q_{6}=\frac{\alpha_{1} b_{m}(1-\varepsilon) x_{2}^{*}}{x_{1}+x_{2}+x_{3}+x_{4}+x_{5}+x_{6}}, \\
& Q_{7}=\frac{\alpha_{2} b_{m} x_{7}^{*}}{x_{1}+x_{2}+x_{3}+x_{4}+x_{5}+x_{6}} .
\end{aligned}
$$

It follows that (60) has a right eigenvector denoted by $v=v_{1}, v_{2}, v_{3}, v_{4}, v_{5}, v_{6}, v_{7}, v_{8}, v_{91}$, where

$$
\begin{aligned}
v_{1}= & \frac{-Q_{5}\left(k_{1} k_{2}+v \psi\right)+\psi\left(Q_{6} k_{1}+v Q_{5}\right) v_{9}}{\left(k_{1} k_{2}-v \psi\right) k_{1}}, \\
v_{2}= & \frac{-\left(Q_{6} k_{1}+v Q_{5}\right) v_{9}}{k_{1} k_{2}-v \psi}, \\
v_{3}= & \frac{Q_{1} v_{9}}{k_{3}}, \\
v_{4}= & \frac{\sigma_{1} Q_{1} v_{9}}{k_{3} k_{4}}, \\
v_{5}= & \frac{\left(1-\sigma_{1}\right) Q_{1} v_{9}}{k_{3} k_{4}}, \\
& v_{6}=\frac{\gamma Q_{1} k_{4}\left(Q_{1}+\left(1-\sigma_{1}\right)\right) v_{9}}{k_{3} k_{4}^{2}}, \\
& v_{7}=\frac{\left(\beta \Lambda_{M} k_{4}^{2} k_{3}+Q_{7} Q_{1} k_{1}\right) v_{9}}{k_{3} k_{4}^{2} \mu_{2}}, \\
& v_{8}=\frac{Q_{7} Q_{1} k_{4}\left(\sigma_{1}+\left(1-\sigma_{1}\right)\right) v_{9}}{k_{3} k_{4}^{2} k_{5}}, \\
& v_{9}=v_{9} .
\end{aligned}
$$

And a left eigenvector given by $w=w_{1}, w_{2}, w_{3}, w_{4}, w_{5}, w_{6}, w_{7}, w_{8}, w_{91}$, where 


$$
\begin{gathered}
w_{1}=\frac{v w_{2}}{k_{1}}, \\
w_{2}=w_{2}, \\
w_{3}=\frac{Q_{7} w_{7}+\gamma w_{6}}{k_{3} k_{4}}, \\
w_{4}=w_{5}=\frac{\gamma w_{6}-Q_{7} w_{7}}{k_{3} k_{4}}, \\
w_{6}=w_{6}, \\
w_{7}=w_{7}, \\
w_{8}=w_{9}=0 .
\end{gathered}
$$

Computation of $a$

$$
\begin{aligned}
& \frac{\partial^{2} f_{1}}{\partial x_{1} \partial x_{9}}=\frac{\alpha_{1} b_{m} x_{1}^{*}}{\left(x_{1}^{*}+x_{2}^{*}\right)^{2}}-\frac{\alpha_{1} b_{m}}{x_{1}^{*}+x_{2}^{*}}, \\
& \frac{\partial^{2} f_{1}}{\partial x_{2} \partial x_{9}}=\frac{\partial^{2} f_{1}}{\partial x_{3} \partial x_{9}}=\frac{\partial^{2} f_{1}}{\partial x_{4} \partial x_{9}}=\frac{\partial^{2} f_{1}}{\partial x_{6} \partial x_{9}}=\frac{\alpha_{1} b_{m} x_{1}^{*}}{\left(x_{1}^{*}+x_{2}^{*}\right)^{2}}, \\
& \frac{\partial^{2} f_{1}}{\partial x_{1} \partial x_{2}}=\frac{k_{1}-\psi}{x_{1}^{*}+x_{2}^{*}}+\frac{2\left(\psi x_{2}^{*}-k_{3} x_{1}^{*}\right)}{\left(x_{1}^{*}+x_{2}^{*}\right)^{3}}, \\
& \frac{\partial^{2} f_{7}}{\partial x_{4} \partial P^{*}}=\frac{\partial^{2} f_{7}}{\partial x_{5} \partial P^{*}}=\frac{\partial^{2} f_{7}}{\partial x_{7} \partial P^{*}}=\frac{x_{7}}{x_{1}^{*}+x_{2}^{*}}, \\
& \frac{\partial^{2} f_{7}}{\partial x_{4} \partial x_{7}}=\frac{\partial^{2} f_{7}}{\partial x_{5} \partial x_{7}}=\frac{\mu_{2}}{\left(x_{1}^{*}+x_{2}^{*}\right)^{2}}-\frac{P^{*}}{x_{1}^{*}+x_{2}^{*}} \text {. } \\
& a=\sum_{k, i, j=1}^{n} v_{k} w_{i} w_{j} \frac{\partial^{2} f_{k}}{\partial x_{i} \partial x_{j}}(0,0) \\
& =v_{1} w_{9} \frac{\alpha_{1} b_{m} x_{1}^{*}}{\left(x_{1}^{*}+x_{2}^{*}\right)^{2}}\left(w_{1}+w_{2}+w_{3}+w_{4}+w_{5}+w_{6}\right) \\
& -v_{1} w_{2}\left(w_{9} \frac{\alpha_{1} b_{m}}{x_{1}^{*}+x_{2}^{*}}+w_{2}\left(\frac{k_{3}+\psi}{x_{1}^{*}+x_{2}^{*}}+\frac{2 \psi x_{2}^{*}+k_{3} x_{1}^{*}}{\left(x_{1}^{*}+x_{2}^{*}\right)^{3}}\right)\right) \\
& -v_{7} w_{7}\left(w_{4}+w_{5}\right)\left(\frac{\mu_{2}}{\left(x_{1}^{*}+x_{2}^{*}\right)^{2}}-\frac{P^{*}}{x_{1}^{*}+x_{2}^{*}}\right) \text {. } \\
& b=\sum_{k, i, j=1}^{n} v_{k} w_{i} \frac{\partial^{2} f_{k}}{\partial x_{i} \partial P^{*}}=v_{7}\left(w_{4}+w_{5}+w_{7}\right)\left(\frac{x_{7}^{*}}{x_{1}^{*}+x_{2}^{*}}\right) .
\end{aligned}
$$

\subsection{Vaccine Impact Analysis}

Vaccine was believed to confer life-long immunity until 1990s. This was the norm as it was approximately correct for most available vaccine for infectious children diseases. But most vaccines used for combating adult infectious diseases today are defective and thus immunity conferred on the recipients wane 
with time. It is expected that the future Chikv vaccine will also be defective and hence the need to assess its effectiveness in $R_{C}$ a community. In this paper, the vaccine impact analysis is done by differentiating effective reproductive number with respect to the proportion $p$ of susceptible individuals vaccinated at equilibrium, according to [32], $\left(p=\frac{V_{H}^{*}}{N_{H}^{*}}\right)$ i.e., $\frac{\partial R_{C}}{\partial p}=\frac{R_{C} \varepsilon}{1(1-p \varepsilon)}$, i.e. since $0<\varepsilon<1$ we have that $\frac{\partial R_{C}}{\partial p}<0$, hence $R_{C}$ is a decreasing function of $p$. This means that a vaccination program with $p>0$ and $\varepsilon>0$ at equilibrium, the future vaccine will have a positive impact. Besides, there exist a $p_{C}$ such that $R_{C}\left(p_{C}\right)=1$ given by $\frac{1}{\varepsilon}\left(1-\frac{1}{R_{C}}\right)$ and for vaccination of proportion of susceptible $p>p_{C}$ the number of new-cases reduces to zero faster than when $p<p_{C}$.

\section{Numerical Simulation}

To further verify the analytical results in the model, the ode 45 code embedded in matlab was used to simulate some parameters of the model. Table 1 provided values of the parameters while initial values of the state variables were chosen arbitrarily. Figures $2(\mathrm{~A})-(\mathrm{D})$ and Figures $3(\mathrm{~A})-(\mathrm{D})$ are simulation of the various model compartments with time. Figure 4 is the simulation of some compartments
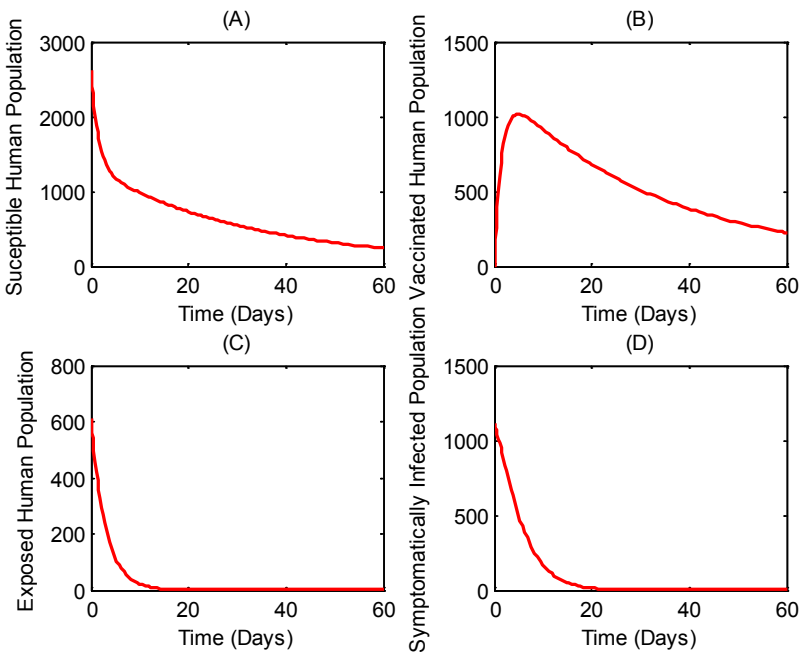

Figure 2. Plot of the various populations with parameters as in Table 1. (A) is the simulation of susceptible human against time, the plot shows that the susceptible human decreases with time due to the proportion that gets infected but slows down after some days, perhaps due to the vaccination and other control measures. (B) is the simulation of the vaccinated compartment. The plot shows a steady increase initially, but began to slope down after few days, this could be due to the fact that a proportion of the class are infectious as the vaccine is imperfect. (C) is the simulation of the exposed compartment with time, the plot shows a steady decline as members become infectious and progress to either the symptomatic or asymptomatic compartment. Finally (D) is the simulation of the symptomatic compartment with time. The plot shows a steady decline and tends to zero after about 20 days. This could be attributed to recovery from the infection. 

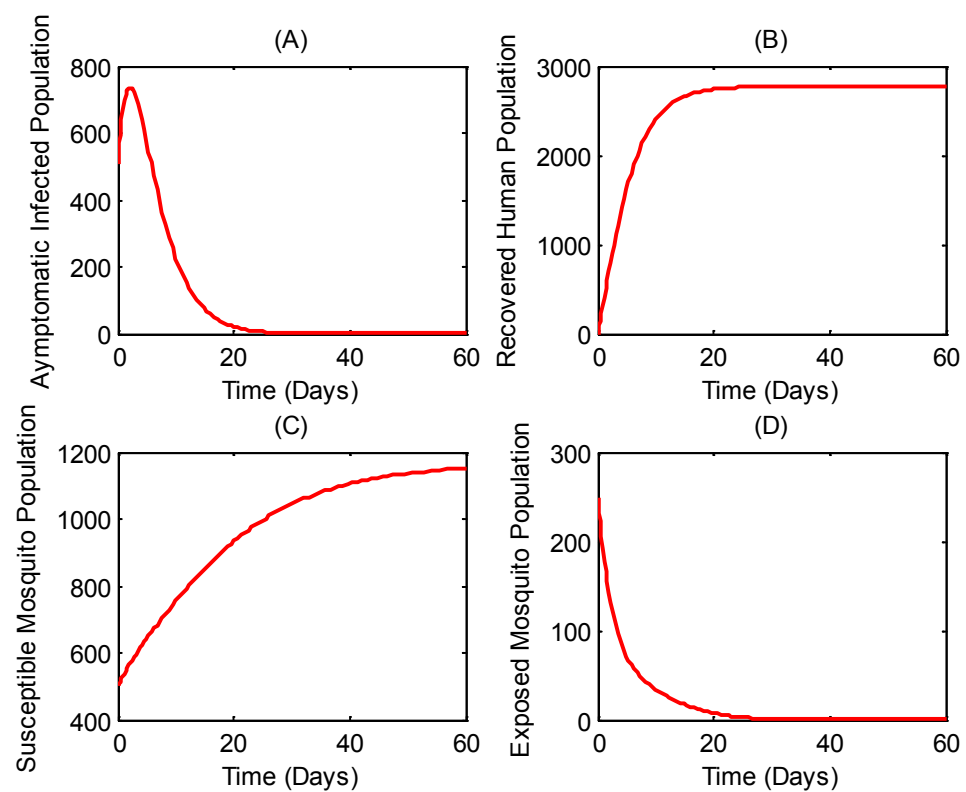

Figure 3. Plot of the various populations with parameters as in Table 1. (A) is the simulation of the asymptomatic infected compartment with time, it shows a sharp increase at the onset of the epidemic, followed by a decline. (B) is the simulation of the recovered compartment with time, it shows a steady increase at the initial time, got to a peak and then remains a constant as time progresses. (C) is the simulation of susceptible mosquito compartment with time. It maintains a steady increase until perhaps due to short life cycle. (D) is the simulation of exposed mosquito compartment with time. The plot shows a steady decline with time as proportion progresses to infected compartment.

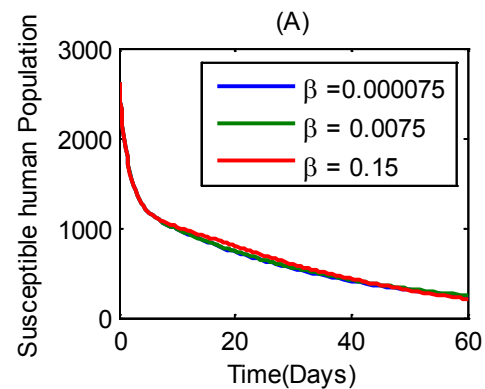

(C)

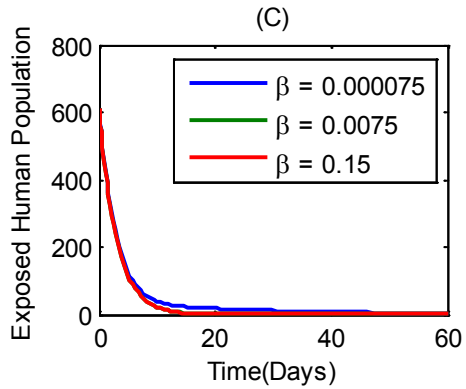

(B)

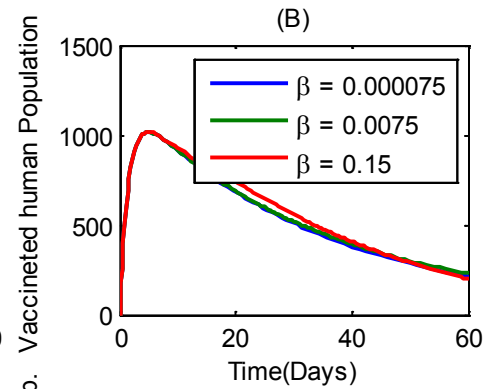

(D)

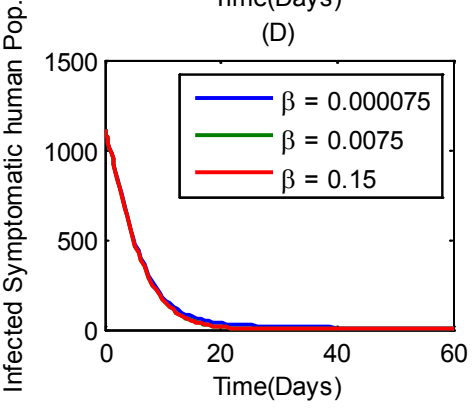

Figure 4. Simulation of the Human populations with varying values of $\beta$. (A) is the effect of the vertical transmission ( $\beta$ ) on the susceptible compartment, while (B), (C) and (D) is the effect on same on the vaccinated, exposed, and symptomatic infected human compartment respectively. It is obvious from the plots that $\beta$ has negligible effect in all the compartments and hence on the transmission of Chikungunya virus according to the model analysis and simulation. 
with various values of the vertical transmission rate $(\beta)$. Figure 5 is a contour plot of the effective basic reproduction number as a function of recruitment rate of susceptible mosquito $\left(\Lambda_{M}\right)$ and vertical transmission rate $(\beta)$ while Figure 6 is the contour plot of effective basic reproductive number with varying values of vaccine efficacy $(\varepsilon)$ and vaccinated proportion. Finally, Figure 7 is a simulation of the new cases of Chikungunya with different values of vaccine efficacy $(\varepsilon)$ and vaccination rate $(v)$. The figures and detailed caption are presented below.

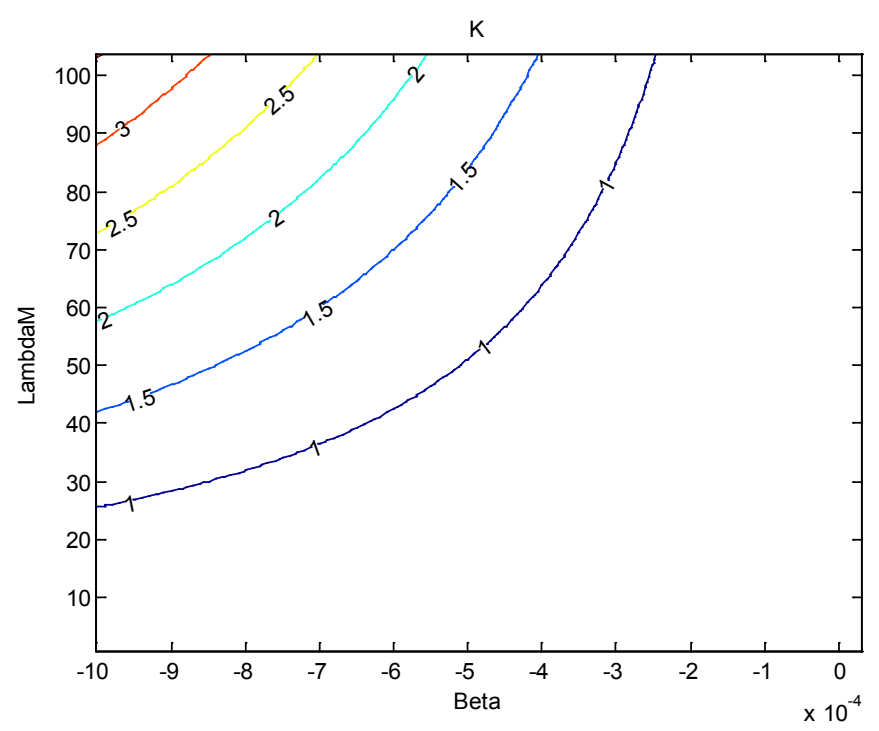

Figure 5. Simulation of the chikv model displaying a contour graph of $R_{C}$ as a function of recruitment rate of susceptible mosquito; and recruitment rate of infected mosquito $(\beta)$ with parameter values as listed in Table 1.

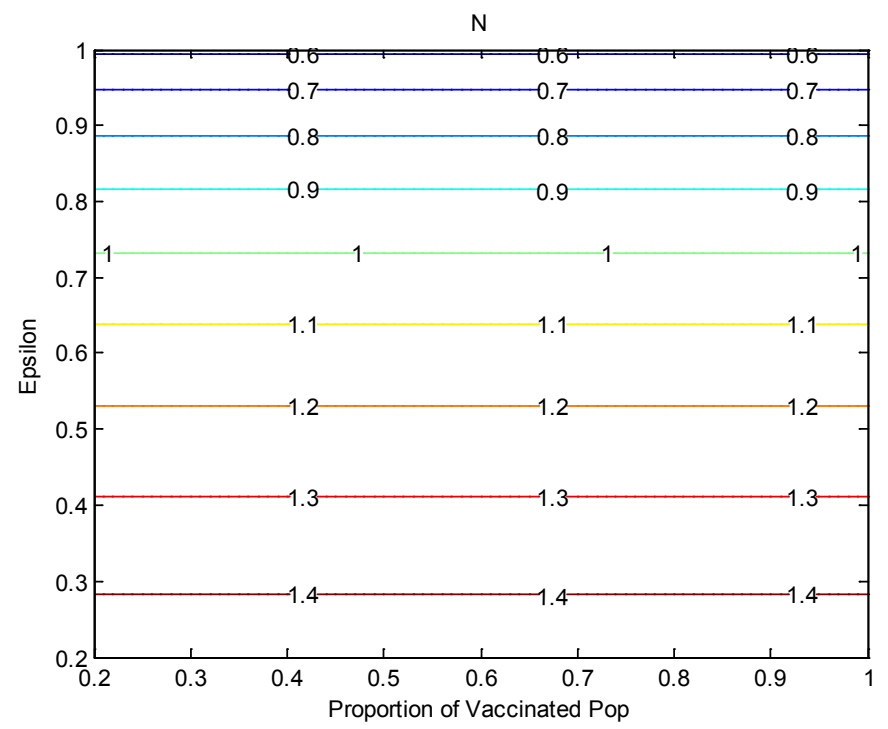

Figure 6. Simulation of the chikv model displaying a contour graph of $\left(R_{c}\right)$ as a function of vaccinated human population and vaccine efficacy $(\varepsilon)$; with parameter values as listed in Table 1. 

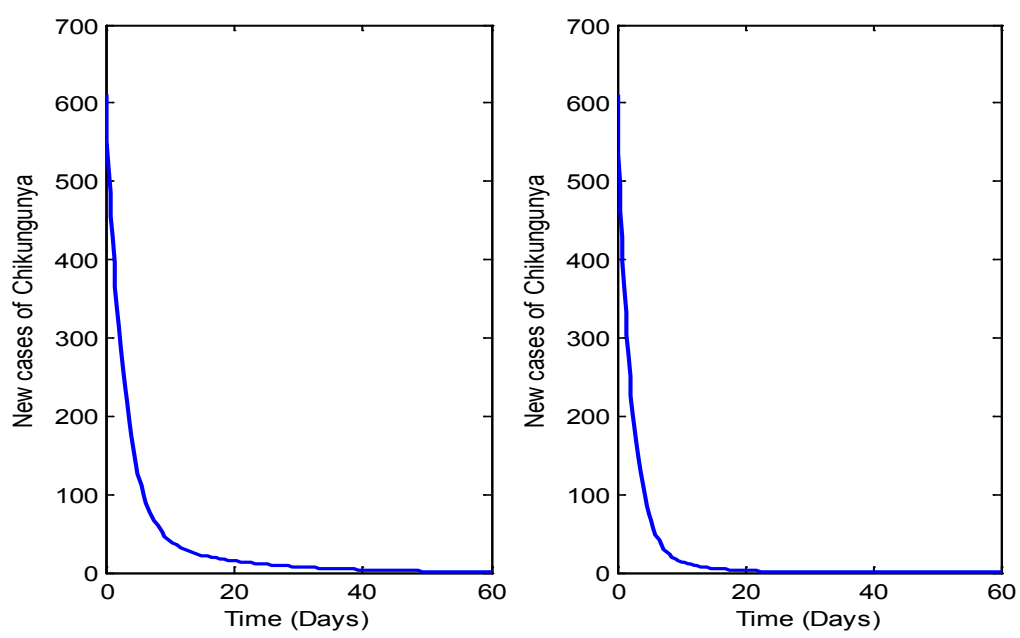

Figure 7. Plot of the new-cases of Chikv model with varying values of vaccine efficacy and, proportion of vaccinated susceptible human population. For (A)

$v=0.6, \varepsilon=0.68, R_{C}=1.0076, P_{C}=0.63156$ For $(\mathrm{B})$

$v=0.63, \varepsilon=0.78, R_{C}=0.8727, P_{C}=0.63156$.

\section{Conclusion}

A deterministic mathematical model for Chikungunya virus dynamics was developed using the standard incidence approach. The model assumed that the offspring of infected mosquito is infected at birth (vertical transmission) and also through blood meal from symptomatically and as-symptomatically infected human (horizontal transmission). For the subhuman population, only horizontal transmission was considered and the virus infection in human is assumed fatal, though with a very low rate. The disease free and endemic equilibrium was obtained and analyzed for both local and global asymptotically stability. The analysis shows that the model undergoes backward bifurcation when the effective basic reproductive number $R_{C} \leq 1$. Numerical simulation of the model shows that the effect of vertical transmission of the mosquito sub-population in the dynamics of the virus is negligible, even when the rate is high as shown in Figures 4(A)-(D). Further, the contour plot of the effective basic reproductive number $R_{C}$ with respect to the vaccine efficacy $\varepsilon$ and the proportion of susceptible vaccinated (Figure 6) gave the rates at which the $R_{C}$ is above, below and equal to unity, this confirms that the use of imperfect vaccine will be effective. Figure 6 also reveals a linear relationship between the effective basic reproductive number and the two parameters in question unlike Figure 5. Also the graph of Chikungunya new case (Figure 7) shows a decrease in new cases with high vaccine efficacy $\varepsilon$ and proportion of vaccinated susceptible $v$. Hence buttressing the point made in Figure 6.

\section{Conflicts of Interest}

The authors declare no conflicts of interest regarding the publication of this paper. 


\section{References}

[1] Robinson, M.C. (1955) An Epidemic of Virus Disease in Southern Province, Tanganyika Territory, in 1952-1953. I. Clinical Features. Transactions of the Royal Society of Tropical Medicine and Hygiene, 49, 28-32. https://doi.org/10.1016/0035-9203(55)90080-8

[2] Vazeille, M., Moutailler, S., Coudrier, D., Rousseaux, C., Khun, H., et al. (2007) Two Chikungunya Isolates from the Outbreak of La Reunion (Indian Ocean) Exhibit Different Patterns of Infection in the Mosquito, Aedes albopictus. PLoS ONE, 2, e1168. https://doi.org/10.1371/journal.pone.0001168

[3] Moore, D.L., Readdy, S., Akinkugbe, F.M., Lee, V.H., David-West, T.S., Causey, O.R. and Carez, D.E. (1974) An Epidemic of Chikungunya Fever at Ibadan, Nigeria, 1969. Annals of Tropical Medicine \& Parasitology, 68, 59-68.

https://doi.org/10.1080/00034983.1974.11686925

[4] World Health Organisation (2017). http://www.who.int/mediacentre/factsheets/fs327/en/

[5] Chandrakant, L., and Pradhan, S.K. (2006) Emergence of Chikungunya Virus in India Subcontinent after 32 Years, a Review. Journal of Vector Borne Diseases, 43, 151-160.

[6] Pialoux, G., Gauze're, B.A., Jaureguiberry, S. and Strobel, M. (2007) Chikungunya, an Epidemic Arbovirosis. The Lancet, 7, 319-327. https://doi.org/10.1016/S1473-3099(07)70107-X

[7] Peterson, L. R., and Powers, A.M. (2016) Chikungunya: Epidemiology. F1000 Research, 5, 82-89.

[8] Reiter, P. (2007) Oviposition, Dispersed and Survival in Aedes aegypti: Implication for the Efficiency of Control Strategies. Vector-Borne Zoonetic Disease, 7, 261-273. https://doi.org/10.1089/vbz.2006.0630

[9] Bonizzoni, M., Gezperi, G., Chen, X. and James, A.A. (2013) The Invasive Mosquito Species Aedes albopictus: Current Knowledge and Future Perspective. Trends in Parasitology, 29, 460-468. https://doi.org/10.1016/j.pt.2013.07.003

[10] Rao, G., Khan, Y.Z. and Chitnis D.S. (2008) Chikungunya Infection in Neonates. Indian Pediatics, 45, 240-244.

[11] Anderson, R.M. and May, R.M. (1991) Infectious Diseases of Humans: Dynamics and Control. Oxford University Press, Oxford.

[12] Hethcote, H.W. (2000) The Mathematics of Infectious Diseases. SIAM Review, 42, 599-653. https://doi.org/10.1137/S0036144500371907

[13] Diekman, O. and Heesterbeck, J.A.P. (2000) Mathematical Epidemiology of Infectious Diseases. Wiley-Blackwell, New York.

[14] Dumont, Y., Chiroleu, F. and Domerg, C. (2008) On a Temporal Model for the Chikungunya Disease: Modeling, Theory and Numerics. Mathematical Biosciences, 213, 80-91. https://doi.org/10.1016/j.mbs.2008.02.008

[15] Moulay, D., Aziz-Alaoui, M.A. and Cadivel, M. (2011) The Chikungunya Disease: Modeling, Vector and Transmission Global Dynamics. Mathematical Biosciences, 229, 50-63. https://doi.org/10.1016/j.mbs.2010.10.008

[16] Ruiz-Moreno, D., Vargas, I.S., Olson, K.E. and Harrington, L.C. (2012) Modeling Dynamic Introduction of Chikungunya Virus in the United States. PLoS Neglected Tropical Disease, 6, e1918. https://doi.org/10.1371/journal.pntd.0001918

[17] Pongsumpun, P. and Sangsawangl, S. (2013) Local Stability Analysis for Age Structural Model of Chikungunya Disease. Journal of Basic and Applied Scientific Re- 
search, 3, 302-312.

[18] Yakob, L. and Clements, A.C.A. (2013) A Mathematical Model of Chikungunya Dynamics and Control: The Major Epidemic on Réunion Island. PLoS ONE, 8, e57448. https://doi.org/10.1371/journal.pone.0057448

[19] Manore, C.A., Hickmann, K.S., Xu, S., Wearing, H.J. and Hyman, J.M. (2014) Comparing Dengue and Chikungunya Emergence and Endemic Transmission in $A$. aegypti and A. albopictus. Journal of Theoretical Biology, 356, 174-191. https://doi.org/10.1016/j.jtbi.2014.04.033

[20] Chitnis, N., Hyman, J.M. and Cushing, J.M. (2008) Determining Important Parameters in the Spread of Malaria through the Sensitivity Analysis of a Mathematical Model. Bulletin of Mathematical Biology, 70, 1272-1296.

https://doi.org/10.1007/s11538-008-9299-0

[21] Dumont, Y. and Chiroleu, F. (2010) Vector Control for the Chikungunya Disease. Mathematical Biosciences and Engineering, 7, 315-348. https://doi.org/10.3934/mbe.2010.7.313

[22] Nur Aida, H., Abu Hassan, A., Nurita, A.T., Che Salmah, M.R. and Norasmah, B. (2008) Population Analysis of Aedes albopictus (Skuse) (Diptera: Culicidae) under Uncontrolled Laboratory Conditions. Tropical Biomedicine, 25, 117-125.

[23] Lahariya, C. and Pradhan, S.K. (2006) Emergence of Chikungunya Virus in Indian Subcontinent after 32 Years: A Review. Journal of Vector Borne Diseases, 43, 151-160.

[24] Delatte, H., Gimonneau, G., Triboire, A. and Fontenille, D. (2009) Influence of Temperature on Immature Development, Survival, Longevity, Fecundity, and Gonotrophic Cycles of Aedes albopictus, Vector of Chikungunya and Dengue in the Indian Ocean. Journal of Medical Entomology, 46, 33-41. https://doi.org/10.1603/033.046.0105

[25] Hethcote, H.W. (1978) An Immunization Model for a Heterogeneous Population. Theoretical Population Biology, 14, 338-349. https://doi.org/10.1016/0040-5809(78)90011-4

[26] Institute of Epidemiology, Disease Control and Research (2017) Chikungunya News Letter. http://www.iedcr.gov.bd/index.php/chikungunya/chikungunya-newsletter

[27] Lakshmikantham, V., Leela, S. and Martynyuk, A.A. (1989) Stability Analysis of Non-Linear Systems. Marcel Dekker, New York.

[28] Castillo-Chavez, C., Blower, S., van den Driessche, P., Kirschner, D. and Yakubu, A.A. (2002) Mathematical Approaches for Emerging and Reemerging Infectious Diseases. Springer-Verlag, New York.

[29] Powell, D.R., Faie, J., Leclaire, R.J., Moore, L.M. and Thompson, D. (2005) Sensitivity Analysis of an Infectious Disease Model. International System Dynamic Conference, Boston, MA.

[30] Castillo-Chavez, C. and Song, B. (2004) Dynamical Models of Tuberculosis and Their Applications. Mathematical Biosciences \& Engineering, 1, 361-404. https://doi.org/10.3934/mbe.2004.1.361

[31] Agosto, F.B., Easley, S., Freeman, K. and Thomas, M. (2016) Mathematical Model of Three Age Structured Transmission Dynamics of Chikungunya Virus. Computational and Mathematical Methods in Medicine, 2016, Article ID: 4320514. https://doi.org/10.1155/2016/4320514

[32] Garba, M.S., Gumel, A.B. and Bakar, A.M.R. (2008) Backward Bifurcation in Dengue Transmission Dynamics. Mathematical Bioscience, 215, 11-25. https://doi.org/10.1016/j.mbs.2008.05.002 


\section{Appendix A}

\section{Castilo-Chaevz and Song [3]}

Consider the following general system of ordinary differential equations with a parameter $\phi$.

$$
\frac{\mathrm{d} x}{\mathrm{~d} t}=f(x, \phi): R^{n} \times R \rightarrow R^{n} \text { and } f \in C^{2}\left(R^{n} \times R\right)
$$

where 0 is an equilibrium point of the system (that is, $f(0, \phi)=0$ for all $\phi$ ) and

(A1) $A=D_{x} f(0,0)=\left(\frac{\partial f_{i}}{d x_{j}}(0,0)\right)$ is the linearization matrix of the system 2.10 around the equilibrium 0 with $\phi$ evaluated at 0 ;

(A2) Zero is a simple eigenvalues of $A$ and other eigenvalues of A have negative real parts;

(A3) Matrix $A$ has a right eigenvector $w$ and left eigenvector $v$ (each corresponding to zero eigenvalues).

Let $f_{k}$ be the $k$ th component of $f$ and

To do this we need the values of $a$ and $b$ given below:

$$
\begin{gathered}
a=\sum_{k, i, j=1}^{n} v_{k} w_{i} w_{j} \frac{\partial^{2} f_{k}}{\partial x_{i} \partial x_{j}}(0,0) \\
b=\sum_{k, i,=1}^{n} v_{k} w_{i} \frac{\partial^{2} f_{k}}{\partial x_{i} \partial x_{\phi}}(0,0)
\end{gathered}
$$

then, the local dynamics of the system around equilibrium point 0 is totally determined by the signs of a and $b$, particularly,

1) $a>0, b>0$, when $\phi<0$ with $|\phi| \ll 1,0$ is locally asymptotically stable and there exists a positive unstable equilibrium; when $0<\phi \ll 1,0$ is unstable and there exists a negative, locally asymptotically stable equilibrium;

2) $a<0, b<0$, when $\phi<0$ with $|\phi| \ll 1,0$ is unstable; when $0<\phi \ll 1,0$ is locally asymptotically stable equilibrium and there exists a positive unstable equilibrium;

3) $a<0, b>0$, when $\phi$ changes from negative to positive, 0 changes its stability from stable to unstable. Correspondingly a negative unstable equilibrium becomes positive and locally asymptotically stable. 